Check for updates

Cite this: Mater. Chem. Front., 2020, 4, 2029

Received 31st March 2020, Accepted 11th May 2020

DOI: $10.1039 / \mathrm{d} 0 \mathrm{qm} 00188 \mathrm{k}$

rsc.li/frontiers-materials

\title{
New exciplex systems composed of triazatruxene donors and $\mathrm{N}$-heteroarene-cored acceptors $\dagger$
}

\author{
Yuan-Cheng Hu, ${ }^{a}$ Zong-Liang Lin, ${ }^{a}$ Tzu-Chien Huang, ${ }^{b}$ Jhih-Wei Lee, ${ }^{b}$ \\ Wei-Chih Wei, ${ }^{a}$ Tzu-Yu Ko, ${ }^{a}$ Chun-Yuan Lo, ${ }^{a}$ Deng-Gao Chen, (DD ${ }^{a}$ \\ Pi-Tai Chou, (D) ${ }^{a}$ Wen-Yi Hung (D) ${ }^{b}$ and Ken-Tsung Wong (D) *ac
}

\begin{abstract}
In this study, three triazatruxene-based molecules Tr-Me, Tr-Ph, and Tr-Tol with methyl, phenyl, and p-tolyl pendant groups, respectively, were intermixed with three acceptors 3P-T2T, 3P-T2P, and 3P-Pyr featuring phenylpyrazole peripherals and triazine, pyrimidine, and pyridine cores, respectively, to generate an array of nine blends to probe the feasibility of formation of an exciplex. The observed redshifted emission compared to those of pristine donor and acceptor films is a good indication of exciplex formation. The exciplex emissions are mainly modulated by the features of $\mathrm{N}$-heteroarene cores of acceptors, while the HOMOs of the donors are less influenced by the substituents. From the screening of these D:A combinations it was concluded that four exciplex-forming blends composed of Tr-Ph and Tr-Tol as donors and 3P-T2T and 3P-T2P as acceptors exhibit promising properties suitable for OLED applications. Among them, a green device employing a Tr-Tol:3P-T2P blend as the emitting layer performed with the best efficiency with a maximum external quantum efficiency $\left(E Q E_{\max }\right)$ of up to 12.8\%. These new exciplex-forming systems were further explored as cohosts for a newly developed fluorescent emitter (DT) ${ }_{2} \mathbf{B T h} 2 \mathrm{CN}$. The Tr-Ph:3P-T2P blend was found to serve as the best host to give a deep-red (EL $\left.\lambda_{\max } 671 \mathrm{~nm}, \mathrm{CIE}(0.68,0.31)\right)$ OLED device with an EQE $\max$ of $5.52 \%$. The data extracted from the transient photoluminescent studies shed light on the emission mechanism which is dominated by Förster energy transfer and simultaneously suffers from competing Dexter energy transfer quenching. Our results highlight the feasibility of using triazatruxene as the donor for exciplex formation as well as the tunability of the emission wavelength of exciplex-forming systems by rational structural modifications of both donors and acceptors.
\end{abstract}

\section{Introduction}

The strategy of efficiently harnessing excitons generated by the recombination of holes and electrons is crucial for highefficiency organic light-emitting diodes (OLEDs). Among many approaches to molecular designs, thanks to the pioneering work reported by Adachi, ${ }^{1}$ organic materials that exhibit thermally activated delayed fluorescence (TADF) are emerging as costcompetitive alternatives to iridium-based phosphorescent emitters ${ }^{2}$ mainly attributable to their capability of achieving $100 \%$ internal quantum efficiency. The fundamental criterion for a molecule to perform TADF is a small energy gap $\left(\Delta E_{\mathrm{ST}}\right)$ between the singlet $\left(\mathrm{S}_{1}\right)$ and triplet $\left(\mathrm{T}_{1}\right)$ excited states. A limited $\Delta E_{\mathrm{ST}}$ allows the triplet

\footnotetext{
${ }^{a}$ Department of Chemistry, National Taiwan University, Taipei, 10617, Taiwan. E-mail: kenwong@ntu.edu.tw

${ }^{b}$ Department of Optoelectronics and Materials Technology, National Taiwan Ocean University, Keelung 202, Taiwan.E-mail: wenhung@mail.ntou.edu.tw ${ }^{c}$ Institute of Atomic and Molecular Science, Academia Sinica, Taipei, 10617, Taiwan

$\dagger$ Electronic supplementary information (ESI) available. See DOI: 10.1039/d0qm00188k
}

excitons to be up-converted to singlet excitons through reverse intersystem crossing (rISC), giving "delayed" fluorescence in addition to intrinsic pump fluorescence. In principle, a small $\Delta E_{\mathrm{ST}}$ can be feasibly realized by a molecule with a low overlap between the highest occupied molecular orbital (HOMO) and the lowest unoccupied molecular orbital (LUMO). Many successful molecular designs have been reported: for example, a bipolar molecule incorporating a twisted $\pi$-bridge ${ }^{3}$ or a $\sigma$-bridge ${ }^{4}$ to separate electron-donor and electron-acceptor blocks. In considering the subtle balance between the limited HOMO/LUMO overlap and $S_{1}$ to $S_{0}$ transition probability, OLED devices employing TADF emitters that can achieve an external quantum efficiency (EQE) of over $36 \%$ have been reported. ${ }^{5-10}$ These exciting results verify the promising potential of organic TADF materials for giving highefficiency OLEDs. Beyond the intramolecular charge transfer (ICT) approach, TADF can also be realized by the recombination of holes and electrons accumulated at the interface of a hole-transporting material (HTM) and an electron-transporting material (ETM) due to the large energy level off-sets. ${ }^{11}$ The HOMO of this interfacial excited state, also known as an exciplex, is located on the HTM, 
whereas the LUMO is positioned at the ETM, leading to a low exchange energy, and therefore a small $\Delta E_{\mathrm{ST}}$ that is suitable for the rISC process. Since the first report of an exciplex showing TADF by Adachi in 2012, ${ }^{12}$ many exciplex-forming systems have been developed to act as the emitting layer (EML) for high-efficiency OLEDs with emissions spanning from blue, ${ }^{13}$ to green ${ }^{14}$ to red, ${ }^{15}$ and even to near infrared. ${ }^{16}$ In addition, exciplex-forming systems can also serve as hosts for fluorescent, ${ }^{17}$ phosphorescent, ${ }^{18}$ and $\mathrm{TADF}^{14}$ dopants for producing superior OLEDs. The versatile functions of exciplex-forming systems suggest promising potential for boosting research activities in material chemistry as well as OLED technology. Among various structures, carbazole-based HTMs are widely utilized as donors in exciplex studies. ${ }^{19-21}$ Similar to carbazole, triazatruxene with a more extended coplanar skeleton has been the core structure for designing good HTMs. ${ }^{22}$ More recently, triazatruxene was reported as serving as a donor moiety for a blue TADF material achieving a record-high performance. ${ }^{23}$ Triazatruxene derivatives also showed other promising opto electronic applications, such as perovskite solar cells ${ }^{24}$ and organic field-effect transistors. ${ }^{25}$ However, to the best of our knowledge, triazatruxene has not been explored for a study of exciplex formation. This triggered our motivation to examine triazatruxene-based HTMs as donors for exciplex-forming systems. Many electron-deficient materials have been verified as good ETMs for exciplexes. In particular, triazine-centered materials are among the best ETMs for giving high-efficiency exciplex-based OLEDs. ${ }^{26}$ In this study, a previously reported ETM, 3P-T2T ${ }^{27}$ with a triazine core equipped with phenylpyrazole peripherals was selected as a model acceptor for blending with triazatruxene-cored HTMs. We envision that the replacement of the central N-heteroarene core from the triazine of 3P-T2T by the pyrimidine of 3P-T2P or the pyridine of 3P-Pyr is a feasible strategy for tuning the electronic properties of ETMs. In conjunction with the 3 different pendant groups (methyl, phenyl, and $p$-tolyl) of triazatruxene, nine possible exciplex-forming blends were characterized with various photophysical methods. Six new exciplex-forming systems were identified for further OLED applications. Among these D:A combinations, a device employing a blend of triazatruxene with a $p$-tolyl substituent (Tr-Tol) and 3P-T2P performed with the best efficiency with a maximum external quantum efficiency $\left(\mathrm{EQE}_{\max }\right)$ of up to $12.8 \%$. These D:A blends were further explored as the host for a tailor-made fluorescent emitter, (DT) $)_{2} \mathbf{B T h} \mathbf{2 C N}$, to give deep-red OLEDs. An OLED device with EL $\lambda_{\max } 671 \mathrm{~nm}, \mathrm{CIE}(0.68,0.31)$ and an $\mathrm{EQE}_{\max }$ of $5.52 \%$ was achieved by using the Tr-Ph:3P-T2P blend as the cohost. Our results highlight the feasibility of tuning the features (emission wavelength and efficiency) of exciplex-forming systems by rational structural modifications that eventually lead to the best D:A blend for OLEDs.

\section{Results and discussions}

The molecular structures and synthetic pathways of the triazatruxenes, 3P-T2T, 3P-T2P, 3P-Pyr, and (DT) ${ }_{2} \mathbf{B T h} 2 \mathbf{C N}$ are depicted in Scheme 1. $\mathbf{T r}-\mathbf{M e}^{28}$ and $\mathbf{T r}-\mathbf{P h}^{29}$ have been reported previously, while Tr-Tol was synthesized by a one-step reaction from the
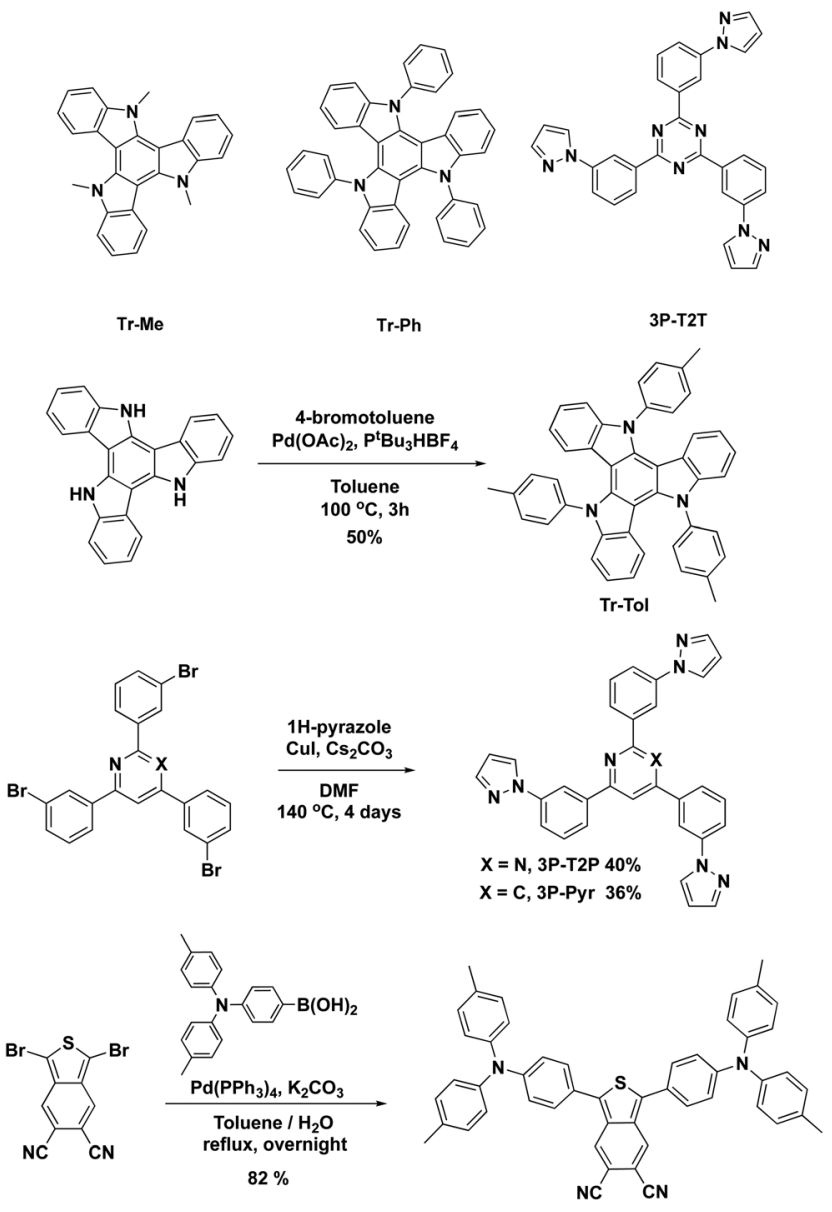

(DT) ${ }_{2} \mathrm{BTh} 2 \mathrm{CN}$

Scheme 1 Molecular structures and synthetic pathways of the triazatruxenes, 3P-T2T, 3P-T2P, 3P-Pyr, and (DT) ${ }_{2}$ BTh2CN.

parent triazatruxene ${ }^{30}$ and 4-iodotoluene by a Pd-catalyzed Hartwig-Buchwald cross-coupling reaction. The model acceptor 3P-T2T was reported in our previous work, ${ }^{27}$ while 3P-T2P and 3P-Pyr were prepared by utilizing a Cu-catalyzed Ullmann crosscoupling reaction of 2,4,6-tris(3-bromophenyl)pyrimidine ${ }^{31}$ and 2,4,6-tris(3-bromophenyl)pyridine ${ }^{32}$ with pyrazole, respectively. In addition, the new fluorescent emitter (DT) ${ }_{2} \mathbf{B T h} 2 \mathbf{C N}$ was synthesized by a one-step Suzuki-Miyaura reaction of reported 1,3-dibromobenzo[c] thiophene-5,6-dicarbonitrile ${ }^{33}$ and [4-[bis(4-methylphenyl)amino]phenyl]boronic acid. All newly synthesized molecules have been characterized with satisfactory spectroscopic data (see ESI $\dagger$ ).

The thermal stability of the material plays a crucial role in the fabrication of OLED devices with a vacuum-deposition process, which is analyzed by thermogravimetric analysis (TGA) under nitrogen. The new triazatruxene Tr-Tol exhibits a reliable thermal stability with a decomposition temperature relative to $5 \%$ weight loss $\left(T_{\mathrm{d}_{95}}\right)$ of $369{ }^{\circ} \mathrm{C}$, which is higher than those of Tr-Me or Tr-Ph reported in the literature, ${ }^{28,29}$ mainly due to the higher molecular weight. The acceptors 3P-T2P and 3P-Pyr, on the other hand, show similar $T_{\mathrm{d}_{95}}$ of 340 and $339{ }^{\circ} \mathrm{C}$, respectively. The thermal stability of 3P-T2T $\left(352{ }^{\circ} \mathrm{C}\right)^{27}$ is relatively higher than those of 3P-T2P 
or 3P-Pyr, which could be attributed to the higher molecular planarity due to the reduced steric interactions between peripheral aryl substitutions and the triazine core. The reduction of the nitrogen atom of the central heteroarene core leads 3P-T2P and 3P-Pyr to suffer from adjacent $\mathrm{C}-\mathrm{H} / \mathrm{C}-\mathrm{H}$ bond repulsions, consequently giving them slightly twisted molecular conformations as well as decreased thermal stability. Nevertheless, from the aspect of optoelectronic applications, these materials possessed sufficiently high thermal stabilities, making them reliable for vacuumdeposition without the risk of decomposition during the hightemperature evaporation. The morphological stabilities of Tr-Tol, 3P-T2P and 3P-Pyr were examined with a differential scanning calorimeter (DSC). The observed glass transition temperature $\left(T_{\mathrm{g}}\right)$ was $64{ }^{\circ} \mathrm{C}$ for 3P-T2P and $67{ }^{\circ} \mathrm{C}$ for 3P-Pyr, whereas there was no phase transition detected for Tr-Tol up to $350{ }^{\circ} \mathrm{C}$.

Cyclic voltammetry was utilized to probe the electrochemical properties. The redox potentials of Tr-Me, Tr-Ph, Tr-Tol, 3P-T2T, 3P-T2P and 3P-Pyr were respectively measured and recorded with reference to a ferrocene/ferrocenium $\left(\mathrm{Fc} / \mathrm{Fc}^{+}\right)$redox couple, as shown in Fig. S1 (ESI $\dagger$ ). A reversible oxidation potential at 0.27, 0.37 and $0.35 \mathrm{~V}$ was observed for Tr-Me, Tr-Ph and Tr-Tol, respectively. Obviously, the electronic properties of triazatruxene can be modulated with the structural feature of the $N$-substitution, in which the methyl-substituted Tr-Me shows a lower oxidation potential compared to those of the aryl-substituted counterparts Tr-Ph and Tr-Tol. There is no surprise that Tr-Tol exhibits a slightly lower oxidation potential than $\mathbf{T r}-\mathbf{P h}$ due to the electron-richer p-tolyl groups. With reference to the $\mathrm{Fc} / \mathrm{Fc}^{+}$redox couple, the HOMO energy levels were estimated as $-5.07 \mathrm{eV}$ (Tr-Me), $-5.17 \mathrm{eV}$ (Tr-Ph), and $-5.15 \mathrm{eV}$ (Tr-Tol). The respective ionization potentials of Tr-Me, Tr-Ph and Tr-Tol solid films were measured by AC-2 to be $-4.96,-5.26$ and $-5.16 \mathrm{eV}$, respectively (Fig. S2 in $\mathrm{ESI} \dagger$ ). The trend observed by AC- 2 measurement agrees with the results from $\mathrm{CV}$ measurement. In contrast to the triazatruxenebased donors, there was a reversible reduction potential at -1.82 and $-2.15 \mathrm{~V}$ for the N-heteroarene-cored acceptors 3P-T2T and 3P-T2P, respectively, while an irreversible reduction potential at $-2.43 \mathrm{~V}$ was observed for 3P-Pyr. Apparently, the number of nitrogen atoms embedded in the $\mathrm{N}$-heteroarene core strongly governs the reduction behavior, in which the most electrondeficient triazine core of 3P-T2T shows a better propensity to be reduced compared to those of 3P-T2P or 3P-Pyr. The LUMO energy levels of 3P-T2T, 3P-T2P and 3P-Pyr were estimated to be $-2.98,-2.65$ and $-2.37 \mathrm{eV}$, respectively, which are in line with the degree of electron deficiency in their central N-heteroarene core. Interestingly, the difference in the HOMO energy levels between triazatruxene-based donors is relatively small, whereas the shift in LUMO energy level is more apparent in N-heteroarenecored acceptors. Therefore, we could reasonably speculate that the exciplex energy upon combining triazatruxene donors and $\mathrm{N}$-heteroarene acceptors would be tuned mainly by the acceptor counterpart.

The photophysical behaviors of triazatruxene-based donors were studied by UV-vis absorption and photoluminescence (PL) spectroscopy, as shown in Fig. 1a. The data are summarized in Table 1. In diluted toluene solution at room temperature, the absorption spectra of Tr-Ph and Tr-Tol corresponding to the $\pi-\pi^{*}$ transition are similar, with a maximum absorption peak $\left(\lambda_{\text {max }}\right)$ centered around $317 \mathrm{~nm}$, which is blue-shifted compared to that $\left(\lambda_{\max }=324 \mathrm{~nm}\right)$ of Tr-Me. In addition, two weak absorption peaks were observed at $332 \mathrm{~nm}$ and $350 \mathrm{~nm}$ for Tr-Ph and Tr-Tol, respectively, whereas Tr-Me shows slightly red-shifted absorptions at 337 and $354 \mathrm{~nm}$, which are also ascribed to the $\pi-\pi^{*}$ transition in the conjugated $\pi$-electron system. ${ }^{28}$ The PL spectra of $\mathbf{T r}-\mathbf{P h}$ and Tr-Tol show a maximum peak (PL $\lambda_{\text {max }}$ ) centered at $391 \mathrm{~nm}$, while the PL $\lambda_{\max }$ of Tr-Me is red-shifted to $396 \mathrm{~nm}$. The slightly red-shifted emission of Tr-Me from Tr-Ph is in line with the results reported in the literature. $^{28,29}$ The absorption and emission spectra of the vacuum-deposited films of Tr-Me, Tr-Ph and Tr-Tol were acquired, as shown in Fig. S3a (ESI $\dagger$ ). Obviously, compared to those of $\mathbf{T r}-\mathbf{P h}$ and $\mathbf{T r}$-Tol, the absorption spectrum of $\mathbf{T r}-\mathbf{M e}$ film exhibits a significant red-shift and broad features, indicating strong intermolecular interactions in the solid state. In addition, all of these triazatruxenes show emissions with vibronic and redshifted characteristics compared to those observed in dilute toluene solution. Parallel to the trend observed in the absorption spectra, the PL spectrum of Tr-Me shows an emission maximum centered at $414 \mathrm{~nm}$, which is significantly red-shifted compared to those of Tr-Ph and Tr-Tol, implying a much stronger propensity for intermolecular aggregations in Tr-Me neat film than in Tr-Ph or Tr-Tol films due to the smaller methyl groups. The strong (a)

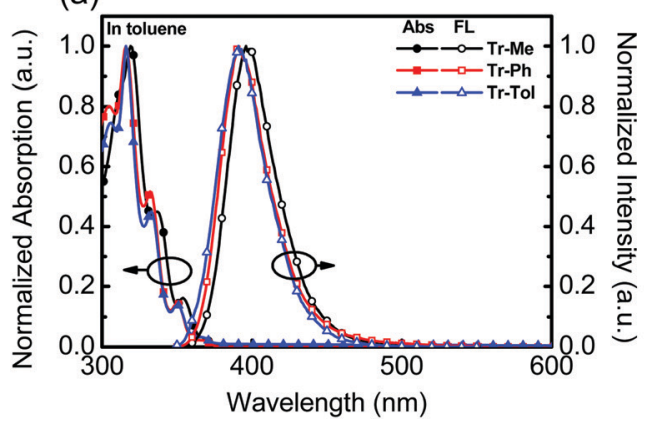

(b)

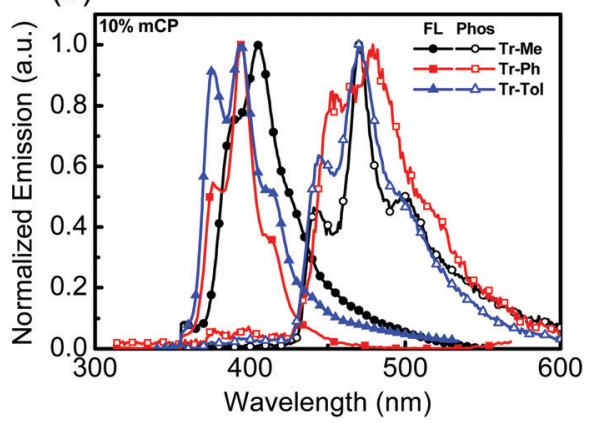

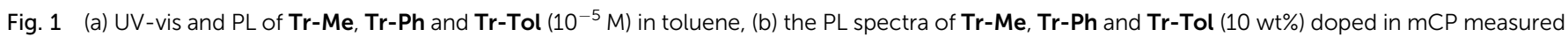
at room temperature and $77 \mathrm{~K}$. 
Table 1 Physical properties of donors Tr-Me, Tr-Ph and Tr-Tol, and acceptors 3P-T2T, 3P-T2P and 3P-Pyr

\begin{tabular}{|c|c|c|c|c|c|c|c|c|c|}
\hline Molecule & $\begin{array}{l}\lambda_{\max }^{a} \text { sol./ } \\
\text { film (nm) }\end{array}$ & $\begin{array}{l}\mathrm{PL} \lambda_{\max }{ }^{a} \mathrm{sol} . / \mathrm{film} / \\
\text { doped film }(\mathrm{nm})\end{array}$ & $\begin{array}{l}\mathrm{PL}_{\mathrm{onset}} \mathrm{nm} \\
(\mathrm{eV})^{d}\end{array}$ & $\begin{array}{l}\text { Phos }_{\text {onset }} \mathrm{nm} \\
(\mathrm{eV})^{e}\end{array}$ & $\begin{array}{l}E_{\mathrm{g}}(\mathrm{sol} .) \\
(\mathrm{eV})\end{array}$ & $\begin{array}{l}\text { HOMO } \\
(\mathrm{eV})\end{array}$ & $\begin{array}{l}\text { LUMO } \\
(\mathrm{eV})\end{array}$ & $\mathrm{IP}(\mathrm{eV})^{f}$ & $T_{\mathrm{d}}\left({ }^{\circ} \mathrm{C}\right)$ \\
\hline Tr-Me & $319,337,354 / 325$ & $396 / 414 / 406$ & $371(3.35)$ & $429(2.90)$ & 3.37 & $-5.07^{b}$ & $-1.70^{c}$ & -4.96 & 327 \\
\hline Tr-Ph & $316,332,350 / 319$ & $391 / 397 / 394$ & $364(3.41)$ & $429(2.90)$ & 3.31 & $-5.17^{b}$ & $-1.86^{c}$ & -5.26 & 363 \\
\hline Tr-Tol & $316,332,350 / 319$ & $391 / 394 / 394$ & $364(3.41)$ & $429(2.90)$ & 3.31 & $-5.15^{b}$ & $-1.84^{c}$ & -5.16 & 369 \\
\hline 3P-T2T & $267 / 268$ & $397 / 419 / 440$ & $395(3.15)$ & $425(2.92)$ & 3.58 & $-6.56^{c}$ & $-2.98^{b}$ & $\mathrm{~N} / \mathrm{A}^{g}$ & $352^{h}$ \\
\hline 3P-T2P & $261 / 259$ & $361 / 380,489 / 434$ & $379(3.28)$ & $430(2.89)$ & 3.50 & $-6.15^{c}$ & $-2.65^{b}$ & N/A & 340 \\
\hline 3P-Pyr & $258 / 259$ & $367 / 367 / 396$ & $349(3.56)$ & $430(2.89)$ & 3.63 & $-6.00^{c}$ & $-2.37^{b}$ & N/A & 339 \\
\hline
\end{tabular}

${ }^{a}$ Measured in toluene at a concentration of about $10^{-5}$ M. ${ }^{b}$ Calculated from potential $v s$. ferrocene/ferrocenium redox couple. ${ }^{c}$ Calculated from the difference between HOMO/LUMO and corresponding optical bandgap. ${ }^{d}$ Estimated from the onset of emission spectra from $10 \%$ materials doped in $\mathrm{mCP}$ at room temperature. ${ }^{e}$ Estimated from the onset of emission spectra from the films of $10 \mathrm{wt} \%$ materials doped in mCP at $77 \mathrm{~K}$. ${ }^{f}$ Measured from AC-2. ${ }^{g}$ Not available. ${ }^{h}$ Reported value.

aggregations render the determination of the energy of the triplet excited state difficult. Therefore, the PL spectra of Tr-Me, Tr-Ph and Tr-Tol (10 wt \%) doped in $\mathrm{mCP}$ were investigated, as shown in Fig. 1b. PL spectra without long wavelength tailing were detected, indicating the suppressed intermolecular interaction, as mCP was employed as a solid matrix. However, a slight redshift in PL $\lambda_{\max }(405 \mathrm{~nm})$ relative to $393 \mathrm{~nm}$ for both $\mathbf{T r}-\mathrm{Ph}$ and Tr-Tol was still observed. Accordingly, the corresponding energies of the singlet state estimated from the emission onset are 3.35, 3.41, $3.41 \mathrm{eV}$ for Tr-Me, Tr-Ph and Tr-Tol, respectively. The phosphorescence spectra of Tr-Me, Tr-Ph and Tr-Tol doped films were acquired at $77 \mathrm{~K}$ (Fig. 1b). Therefore, the triplet energy was estimated to be $2.90 \mathrm{eV}$ for all these triazatruxenebased donors by the onset $(429 \mathrm{~nm})$ of phosphorescence. Apparently, the $N$-phenyl or $N$-p-tolyl rings are twisted with respect to the triazatruxene core, thereby preventing intermolecular $\pi-\pi$ interactions in $\mathbf{T r}-\mathbf{P h}$ and $\mathbf{T r}-\mathbf{T o l}$ molecules. Whereas, lacking intrinsic structural congestion, Tr-Me molecules form severe self-aggregations that implies a poor tendency for forming a uniform film mixed with acceptor materials.

The photophysical behaviors of N-heteroarene-cored acceptors were also studied by UV-vis absorption and PL spectroscopy, as shown in Fig. 2a. The data are summarized in Table 1. The absorption spectra of 3P-T2P and 3P-Pyr show similar results with the absorption peaks at around 259 and $325 \mathrm{~nm}$, which could be attributed to the $\pi-\pi^{*}$ transition and $\mathrm{n}-\pi^{*}$ transition, ${ }^{34,35}$ respectively. The absorption edge can be unambiguously assigned to determine the optical energy gap to be $3.58,3.50$ and $3.63 \mathrm{eV}$ for 3P-T2T, 3P-T2P and 3P-Pyr, respectively. The emission with the PL $\lambda_{\max }$ of 3P-T2P and 3P-Pyr in dilute $\mathrm{CH}_{2} \mathrm{Cl}_{2}$ solution is detected at $360 \mathrm{~nm}$, which is much blue-shifted compared to that of 3P-T2T (395 $\mathrm{nm}$ ) reported previously. ${ }^{27}$ The absorptions (Fig. S3b, ESI $\dagger$ ) of 3P-T2P and 3P-Pyr films are similar with $\lambda_{\max }$ at around $259 \mathrm{~nm}$, which is slightly blue-shifted from the $268 \mathrm{~nm}$ of 3P-T2P film. ${ }^{27}$ Apart from 3P-T2T and 3P-Pyr, which show single emission peaks centered at 420 and $367 \mathrm{~nm}$, respectively, in their emission spectra of neat films, the emission spectrum of 3P-T2P shows a weak emission at $380 \mathrm{~nm}$ corresponding to its typical molecular chromophore and a strong and broad emission centered at $490 \mathrm{~nm}$ ascribed to the excimer emission. The emission spectra of 3P-T2P, 3P-T2P and 3P-Pyr (10 wt\%) blended in mCP were examined, as shown in Fig. $2 \mathrm{~b}$. The lower energy emission of 3P-T2P disappeared when it served as a guest dopant dispersed in an MCP host, verifying the possibility of forming excimer emission in pristine film. The respective PL $\lambda_{\max }$ of 3P-T2P, 3P-T2P and 3P-Pyr blended films are distinguishable, indicating the effects of the electronaccepting power of the central $\mathrm{N}$-heteroarenes and molecular rigidity on the singlet energy. The higher electron-withdrawing propensity of the triazine core together with the lack of $\mathrm{C}-\mathrm{H} /$ C-H steric interactions leads 3P-T2T to have a red-shifted $\lambda_{\max }$ of $395 \mathrm{~nm}$ as compared to the $379 \mathrm{~nm}$ of 3P-T2P and $349 \mathrm{~nm}$ of 3P-Pyr. The triplet energies of 3P-T2T, 3P-T2P and 3P-Pyr were determined to be $2.92,2.89$ and $2.89 \mathrm{eV}$, respectively, based on the onset energy of the phosphorescence spectra (Fig. 2b) obtained at $77 \mathrm{~K}$. (a)

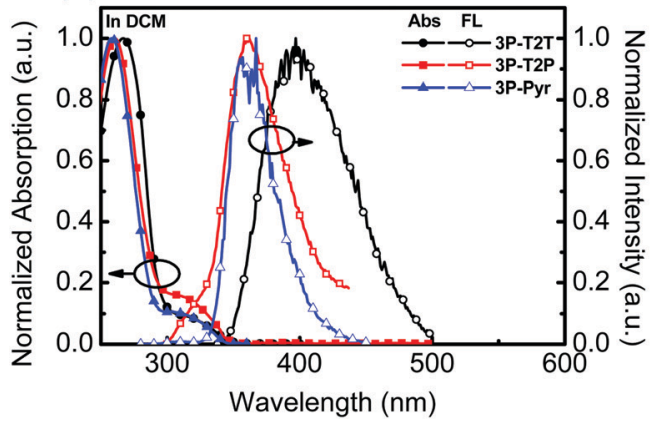

(b)

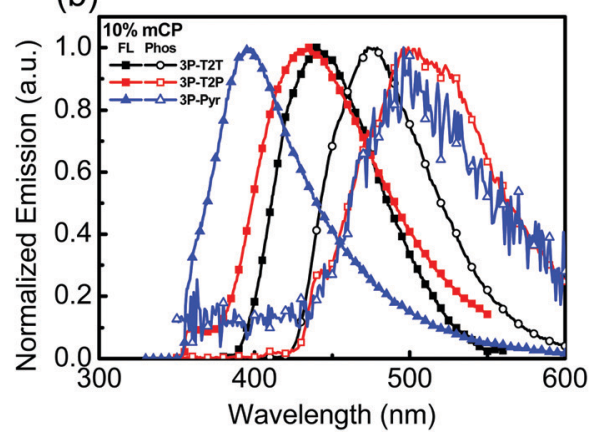

Fig. 2 (a) UV-vis and PL of 3P-T2T, 3P-T2P and 3P-Pyr $\left(10^{-5} \mathrm{M}\right.$ ) in $\mathrm{CH}_{2} \mathrm{Cl}_{2}$, (b) the PL spectra of 3P-T2T, 3P-T2P and 3P-Pyr (10 wt\%) doped in mCP measured at room temperature and $77 \mathrm{~K}$. 
(a)

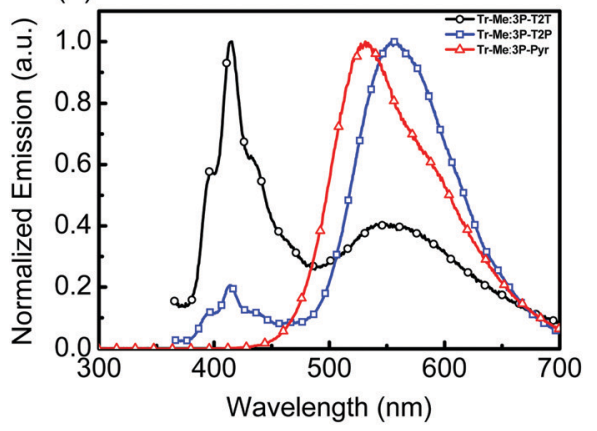

(b)

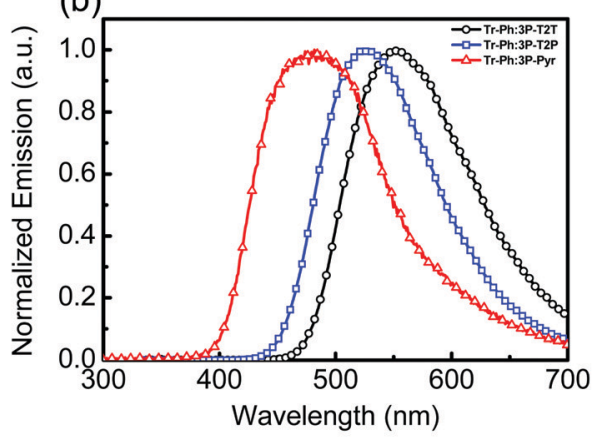

(c)

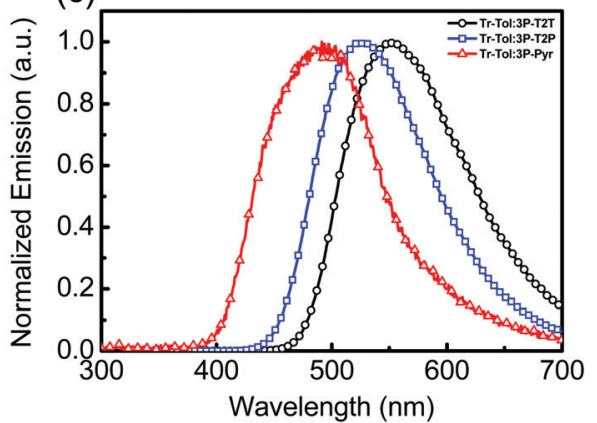

Fig. 3 Emission spectra of (a) Tr-Me:acceptor, (b) Tr-Ph:acceptor and (c) Tr-Tol:acceptor intermixed films.

To explore the formation of exciplexes using Tr-Me, Tr-Ph and Tr-Tol as donors and 3P-T2T, 3P-T2P and 3P-Pyr as acceptors, an array of 9 vacuum-processed D:A $(1: 1)$ blends was prepared. The absorption spectra (Fig. S4, ESI $\dagger$ ) of all blended films show strong absorption peaks around 260 and $320 \mathrm{~nm}$, which can be considered to be the linear combination of composing donor and acceptor materials. The corresponding emission spectra are depicted in Fig. 3 and the data are summarized in Table 2. No significant new absorption peaks are found in these blends, indicating the lack of ground state interactions between donors and acceptors. In contrast, the blended films did exhibit distinct emission spectra different from their components. As compared to the individual emission of donor or acceptor, the red-shifted

Table 2 The photophysical characteristics of D:A blended films

\begin{tabular}{|c|c|c|c|c|c|c|c|c|}
\hline \multirow[b]{2}{*}{ Donor } & \multirow[b]{2}{*}{ Acceptor } & \multirow{2}{*}{$\begin{array}{l}\mathrm{PLQY}^{a} \\
(\%)\end{array}$} & \multirow{2}{*}{$\mathrm{PL}_{\max / \text { onset }}(\mathrm{nm})$} & \multirow{2}{*}{$\begin{array}{l}\Delta E_{\mathrm{ST}}{ }^{b} \\
(\mathrm{eV})\end{array}$} & \multicolumn{4}{|c|}{$\mathrm{TRPL}^{c}$} \\
\hline & & & & & $A_{1}$ & $\tau_{1}(\mu \mathrm{s})$ & $A_{2}$ & $\tau_{2}(\mathrm{~ns})$ \\
\hline \multirow[t]{3}{*}{ Tr-Ph } & 3P-T2T & 39 & $553 / 479(2.59)^{d}$ & 0.10 & 0.11 & 1.95 & 0.89 & 33.1 \\
\hline & 3P-T2P & 41 & $526 / 456(2.73)$ & 0.18 & 0.09 & 1.77 & 0.91 & 52.0 \\
\hline & 3P-Pyr & 8 & $480 / 405(3.07)$ & 0.34 & 0.06 & 0.07 & 0.94 & 25.6 \\
\hline \multirow[t]{3}{*}{ Tr-Tol } & 3P-T2T & 33 & $553 / 480(2.59)$ & 0.10 & 0.10 & 1.73 & 0.90 & 25.8 \\
\hline & 3P-T2P & 40 & $525 / 457(2.73)$ & 0.10 & 0.09 & 2.39 & 0.91 & 56.4 \\
\hline & 3P-Pyr & 10 & $491 / 405(3.07)$ & 0.33 & 0.04 & 1.0 & 0.96 & 29.0 \\
\hline
\end{tabular}

${ }^{a}$ The PLQY for the blend were measured with an integrating sphere (Hamamatsu C9920-02). ${ }^{b} \Delta E_{\mathrm{ST}}$ are calculated from the energy difference between the emission onsets of prompt and delayed emissions, as shown in Fig. S6 (ESI). ${ }^{c}$ The TRPL profiles were measured under an ambient atmosphere, and the decay components were fitted with two exponential decay models as $I(\tau)=A_{1} \exp \left(-t / \tau_{1}\right)+A_{2} \exp \left(-t / \tau_{2}\right)$, as shown in Fig. S5 (ESI). ${ }^{d}$ Derived optical energy gap (eV) from $1242.4 / \mathrm{PL}_{\text {onset }}(\mathrm{nm})$. emission of blended film is the signature of forming an exciplex upon photoexcitation. The blends consisting of Tr-Me as donor mixed with acceptors 3P-T2T, 3P-T2P and 3P-Pyr gave emission peaks (Fig. 3a) centered at 551, 557 and $530 \mathrm{~nm}$, respectively. In addition, the PL spectra of Tr-Me:3P-T2T and Tr-Me:3P-T2P blends show the residual emission of Tr-Me, indicating incomplete exciplex formation due to the self-segregation of less sterically hindered Tr-Me together with more coplanar acceptors 3P-T2T and 3P-T2P. However, the emission of individual D or A components disappeared in the Tr-Me:3P-Pyr blend, indicating that the twisted conformation of 3P-Pyr is beneficial for suppressing its self-segregation and also blocking the severe intermolecular interactions of Tr-Me molecules. The Tr-Ph-blended films composed of acceptors 3P-T2T, 3P-T2P and 3P-Pyr show emission (Fig. 3b) centered at 552, 527 and $482 \mathrm{~nm}$, respectively. Finally, the blends formed with Tr-Tol as donor and 3P-T2T, 3P-T2P and 3P-Pyr as respective acceptors give emission peaks (Fig. 3c) centered at 554, 526 and $492 \mathrm{~nm}$, respectively. In general, the Tr-Me-based blends exhibit red-shifted emissions compared to those counterpart blends with Tr-Ph and Tr-Tol as donors due to its higher lying HOMO energy level. Obviously, the energy of exciplex emission, typically correlated with the energy difference between donor (HOMO) and acceptor (LUMO), ${ }^{19}$ is governed mainly by the structural features of $\mathrm{N}$-heteroarene centers. Time-resolved photoluminescence (TRPL) experiments, except for the incomplete exciplex formation of the blends with Tr-Me as donor, were conducted to probe the relaxation processes of these exciplex emissions (Fig. S5, ESI $\dagger$ ). The results are summarized in Table 2. We speculate that the low electron deficiency of 3P-Pyr together with relatively weak donors Tr-Ph and Tr-Tol may not 
induce sufficient polarization for efficient rISC upon photoexcitation. ${ }^{36}$ The pump and delayed fluorescence spectra are provided in Fig. S6 (ESI $\dagger$ ). The prompt emission acquired by an intensified charge couple detector at zero delay time and a gate width of $100 \mathrm{~ns}$ is attributed to fluorescence, while the delayed emission acquired at a delay time of $1 \mu$ s and gate width of $10 \mu \mathrm{s}$ is ascribed to delayed fluorescence. The prompt and delayed emissions were recorded from the emission onsets to calculate the singlet-triplet splitting energy $\left(\Delta E_{\mathrm{ST}}\right)$. Obviously, the large $\Delta E_{\text {ST }}$ of Tr-Ph: 3P-Pyr $(0.34 \mathrm{eV})$ and Tr-Tol: 3P-Pyr $(0.33 \mathrm{eV})$ make it hard to undergo an efficient rISC process that leads to low PLQY. To our delight, the TRPL of the 4 blends consisting of Tr-Ph and Tr-Tol as donors and 3P-T2T and 3P-T2P as acceptors showed rather evident delayed components in a microsecond timescale and moderate PLQYs ranging from 33 to $41 \%$, indicating the possibility of producing better device characteristics due to superior TADF characters.

\section{Device}

To investigate the electroluminescence (EL) from the exciplexes, OLEDs were fabricated with the device architecture ITO/4 wt\% $\mathrm{ReO}_{3}$ :donor $(60 \mathrm{~nm}) /$ donor $(15 \mathrm{~nm}) / \mathrm{D}: \mathrm{A}(1: 1,20 \mathrm{~nm}) /$ acceptor $(10 \mathrm{~nm}) / \mathrm{CN}-\mathrm{T} 2 \mathrm{~T}(40 \mathrm{~nm}) / \mathrm{Liq}(0.5 \mathrm{~nm}) / \mathrm{Al}(100 \mathrm{~nm})$. ITO and $\mathrm{Liq} / \mathrm{Al}$ are the anode and the cathode, respectively (Fig. 4). Due to the severe self-aggregation of Tr-Me, only Tr-Ph and Tr-Tol were selected as donors to blend with acceptors 3P-T2T, 3P-T2P and 3P-Pyr. The mixtures of $\mathrm{D}$ and $\mathrm{A}$ (1:1 weight ratio) were introduced as the EML. The $4 \mathrm{wt} \%$ rhenium oxide $\left(\mathrm{ReO}_{3}\right)$ doped in the donor was used as the hole injection layer (HIL). ${ }^{37}$ The hole mobility of Tr-Ph and Tr-Tol determined by the time-offlight technique is $c a \cdot 10^{-4} \mathrm{~cm}^{2} \mathrm{~V}^{-1} \mathrm{~s}^{-1}$, as shown in Fig. S7 (ESI $\dagger$ ), making donors Tr-Ph and Tr-Tol good hole-transporting layers. A previously reported $3^{\prime}, 3^{\prime \prime \prime}, 3^{\prime \prime \prime \prime \prime}-(1,3,5$-triazine-2,4,6triyl)tris-([1,1'-biphenyl]-3-carbonitrile) $(\mathrm{CN}-\mathrm{T} 2 \mathrm{~T})^{38}$ with a high triplet level $\left(E_{\mathrm{T}}=2.82 \mathrm{eV}\right)$, suitable HOMO/LUMO $(-6.7 /$ $-2.78 \mathrm{eV})$ and high electron mobility $\left(\mu_{\mathrm{e}}=10^{-4} \mathrm{~cm}^{2} \mathrm{~V}^{-1} \mathrm{~s}^{-1}\right.$ order) was selected as the electron-transporting layer. The additional $10 \mathrm{~nm}$ acceptor was introduced as a hole-blocking layer (HBL) to prevent undesirable exciplex formation between donor and CN-T2T.

Fig. 5 depicts the current density-voltage-luminance $(J-V-L)$ characteristics, device efficiencies, and EL spectra. The key characteristics are summarized in Table 3. Fig. 4b shows a schematic energy level of the donors and acceptors as well as the fluorescent emitter used in this work. The HOMO levels of Tr-Ph and Tr-Tol are similar $(-5.17$ and $-5.15 \mathrm{eV})$. However, the LUMO levels of 3P-Pyr $(-2.37 \mathrm{eV})>$ 3P-T2P $(-2.65 \mathrm{eV})>$ 3P-T2T $(-2.98 \mathrm{eV})$ will strongly influence the emissive property of the resultant exciplex. Devices A1 and B1 use the EML where 3P-T2T was selected as the acceptor to blend with $\mathbf{T r}-\mathbf{P h}$ and Tr-Tol, respectively. The electrons are injected from CN-T2T to 3P-T2T without any energy barrier, leading to a low turn-on voltage $\left(V_{\text {on }}\right)$ of $2.0 \mathrm{eV}$ and a high current density. Device $\mathbf{A 1}$ gives a luminance $(L)$ of $84318 \mathrm{~cd} \mathrm{~m}^{-2}$ at $6.0 \mathrm{~V}\left(1309 \mathrm{~mA} \mathrm{~cm}^{-2}\right)$ with an EL peak at $558 \mathrm{~nm}$ and CIE coordinates of $(0.43,0.53)$ and the maximum EQE, current efficiency (CE), and power efficiencies (PE) are 10.3\%, $29.8 \mathrm{~cd} \mathrm{~A}^{-1}$ and $37.5 \mathrm{~lm} \mathrm{~W}^{-1}$. The extremely low driving voltage of $2.7 \mathrm{~V}$ at $1000 \mathrm{~cd} \mathrm{~m}^{-2}$ means that the carriers are injected smoothly into the HOMO/LUMO levels of the exciplex EML without any energy barrier. Device A1 retains an EQE of $9.9 \%$ at a high luminance of $1000 \mathrm{~cd} \mathrm{~m}^{-2}$. In addition, device $\mathbf{A 1}$ exhibits better efficiency than device $\mathbf{B} 1$ that exhibits EQE, CE, and PE of 9.7\%, $27.3 \mathrm{~cd} \mathrm{~A}^{-1}$, and $35.7 \mathrm{~lm} \mathrm{~W}^{-1}$ and CIE coordinates of $(0.44,0.53)$. This result is consistent with the PLQYs measured for the thin films of Tr-Ph:3P-T2T (39\%) and Tr-Tol:3P-T2T (33\%) blends. Obviously, similar to the previously reported results regarding $3 \mathbf{P}-\mathbf{T} 2 \mathbf{T}^{39-41}$ as a good acceptor with other donors for efficient exciplex systems, the triazatruxene-based molecules $\mathbf{T r}-\mathbf{P h}$ and $\mathbf{T r}$ - $\mathbf{T o l}$ are also good donors, which can be further utilized to examine the new exciplex-forming systems with other acceptors.

Devices A2 and B2 using the exciplex-based EML composed of 3P-T2P as acceptor and Tr-Ph and Tr-Tol as donors, respectively, exhibit green emissions, which are blue-shifted compared to those of the devices employing 3P-T2T as an acceptor due to the higher LUMO energy of 3P-T2P. The lower current density and higher $V_{\text {on }}$ for 3P-T2P-based devices are due to the energy barrier $(0.13 \mathrm{eV})$ for the electron injection from CN-T2T to 3P-T2P. Device B2 based on the Tr-Tol:3P-T2P blend as the EML shows the best performance among the devices reported in this work, giving a $V_{\text {on }}$ of $2.2 \mathrm{~V}$ and maximum efficiencies as high as $12.8 \%$ (EQE), $38.3 \mathrm{~cd} \mathrm{~A}^{-1}$ (CE), and $46.3 \mathrm{~lm} \mathrm{~W}^{-1}$ (PE) with CIE coordinates of $(0.35,0.54)$. Correspondingly, the device with the Tr-Ph:3P-T2P blend shows lower maximum efficiencies $\left(10.4 \%, 30.8 \mathrm{~cd} \mathrm{~A} \mathrm{~A}^{-1}, 37.2 \mathrm{~lm} \mathrm{~W}^{-1}\right)$ with CIE coordinates of $(0.33,0.54)$. The superior efficiency obtained in devices $\mathbf{A 2}$ and $\mathbf{B} 2$ agrees with the observed better (a)

\begin{tabular}{c|c|c|c|c|c|c} 
HIL & HTL & EML & HBL & ETL & \\
\hline $60(\mathrm{~nm})$ & 15 & 20 & 10 & 40 &
\end{tabular}

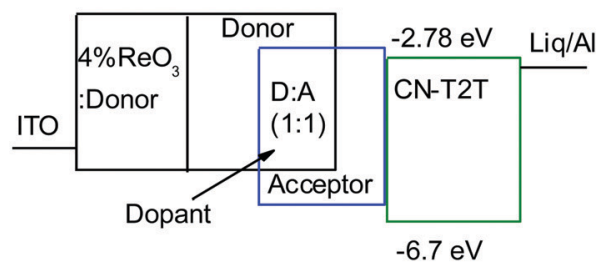

(b)

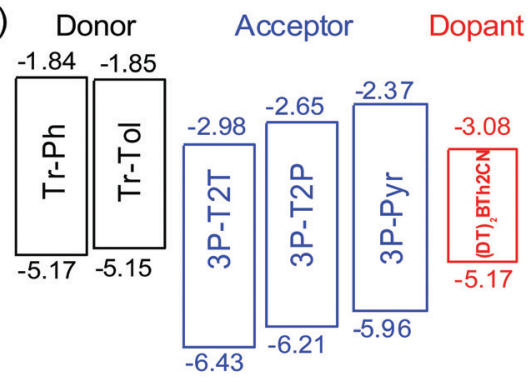

Fig. 4 (a) Schematic exciplex-based OLED structure, and (b) energy levels of donors, acceptors, and fluorescent emitter used in this study. 

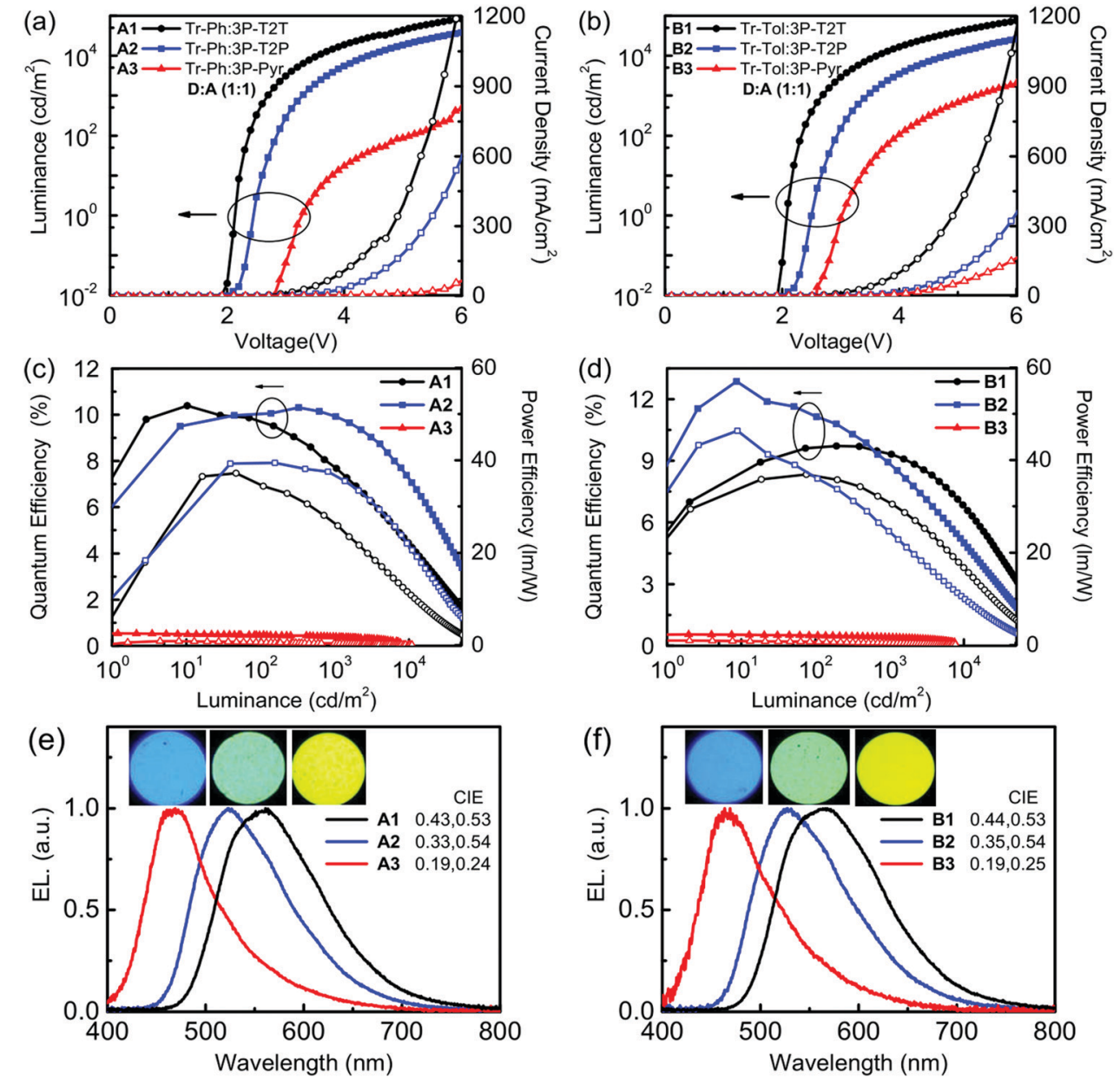

Fig. 5 (a)/(b) Current density-voltage-luminance $(J-V-L)$ characteristics, (c)/(d) external quantum efficiencies (EQE) and power efficiencies (PE) as a function of luminance, and (e)/(f) EL spectra of Tr-Ph and Tr-Tol based exciplex devices.

PLQYs of exciplex-forming systems with 3P-T2P as the acceptor (Table 2). Devices A3 and B3 use 3P-Pyr as an acceptor and Tr-Ph and Tr-Tol as a donor, respectively, as the EML for making exciplexes. The higher LUMO level $(-2.37 \mathrm{eV})$ of 3P-Pyr leads the exciplex to have blue emission with CIE coordinates of $(0.19,0.24-$ 0.25). The low $J-V$ performance and high $V_{\text {on }}$ are because of the larger energy barrier ( $0.41 \mathrm{eV}$, see Fig. $4 \mathrm{a}$ and b) between CN-T2T and 3P-Pyr that impedes the electron injection to the EML. Together with the low fluorescence quantum yield of $8 \%$ for Tr-Ph:3P-Pyr and 10\% for Tr-Tol:3P-Pyr, these give the low EQE $(<1 \%)$ of 3P-Pyr-based blue devices.

The highly efficient exciplex system can be employed as the cohost for improving the efficiencies of OLEDs ${ }^{42}$ because the triplet excitons of the exciplex can be up-converted back to singlets by way of the efficient rISC process. Then, the singlet excitons of the exciplex cohost can transfer to the singlet state of the fluorescent dopant via a Förster energy transfer (FRET) pathway, making high-efficiency fluorescent OLEDs accessible. ${ }^{11}$ To realize an effective FRET, a good spectral overlap between the emission of the host and the absorption of the dopant is required. Fig. 6(a) displays the good overlaps between the absorption spectrum of the newly developed fluorescent dopant $(\mathbf{D T})_{2} \mathbf{B T h} 2 \mathbf{C N}$
(Scheme 1) and the PL spectra of exciplex cohosts (Tr-Ph/Tr-Tol: 3P-T2T and Tr-Ph/Tr-Tol:3P-T2P), ensuring that efficient energy transfer from the exciplex to the dopant is feasible.

The exciplex-forming systems were further exploited as cohosts for a red fluorescent dopant (DT) ${ }_{2} \mathbf{B T h} 2 \mathrm{CN}$. The photophysical and electrochemical properties of $(\mathbf{D T})_{2} \mathbf{B T h} 2 \mathbf{C N}$ are depicted in Fig. S8 (ESI $\dagger$ ), and the data together with the thermal stability are summarized Table S1 (ESI $\dagger$ ). First, the devices employing the best exciplex Tr-Tol:3P-T2P blend as the cohost incorporated with various doping concentrations of (DT) ${ }_{2} \mathbf{B T h} 2 \mathrm{CN}$ were fabricated to optimize the EL performance (Fig. S9, ESI $\dagger$ ). The device doped with 1 wt $\%$ (DT) $)_{2} \mathbf{B T h} 2 \mathbf{C N}$ exhibits maximum EQE and $\mathrm{PE}$ of $5.5 \%$ and $8.4 \mathrm{~lm} \mathrm{~W}^{-1}$, respectively. However, at such a low doping concentration, the EL spectrum shows residual emission from the exciplex cohost with an EL peak at $520 \mathrm{~nm} .{ }^{43}$ The device doped with 5 wt\% (DT) $)_{2}$ BTh2CN displays better energy transfer; however, a weak emission from the exciplex cohost still remains. This device shows the EL emission peak centered at $660 \mathrm{~nm}$ and CIE coordinates of $(0.64,0.35)$, but a lower maximum EQE $(4.39 \%)$ compared to the $1 \mathrm{wt} \%$ doped device. When the doping concentration increases to $10 \mathrm{wt} \%$, the device retains a comparable EQE 
Table 3 Electroluminescence data of OLED devices

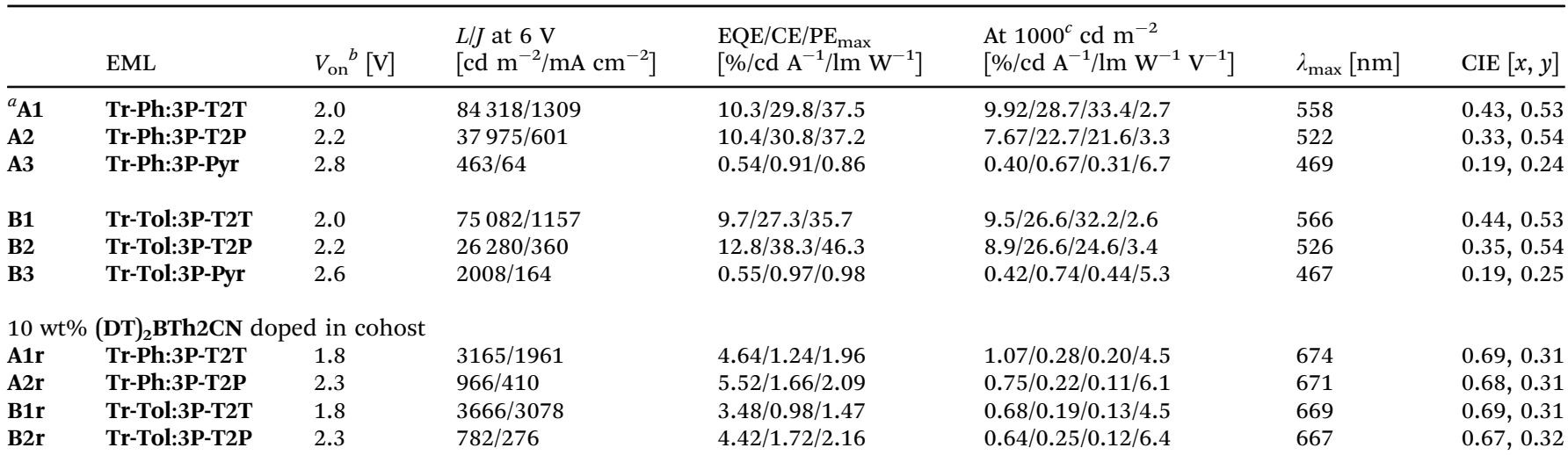

${ }^{a}$ The notation $\mathbf{A}$ and $\mathbf{B}$ indicate the donors Tr-Ph and Tr-Tol, respectively. The notation 1-3 indicate the acceptors 3P-T2T, 3P-T2P and 3P-Pyr, respectively. The notation $\mathbf{r}$ indicates $10 \mathrm{wt} \%(\mathbf{D T})_{2} \mathbf{B T h} 2 \mathbf{C N}$ as dopant. ${ }^{b}$ Turn-on voltage at which emission became detectable. ${ }^{c}$ The values of EQE, CE, PE and driving voltages of device at $1000 \mathrm{~cd} \mathrm{~m}^{-2}$.

(4.42\%) and exhibits EL solely from the dopant but with the peak further red-shifted to $672 \mathrm{~nm}$ and CIE coordinates of $(0.67,0.32)$, indicating complete energy transfer. The color-shifting is because of the intermolecular interactions and strong local polarization field. ${ }^{44}$ The reduced device efficiency found in the devices with higher doping concentrations of (DT) $)_{2} \mathbf{B T h} 2 \mathbf{C N}$ can be plausibly ascribed to the triplet-triplet Dexter energy transfer that leads to direct exciton quenching by the non-emissive triplets of the fluorescent dopant. To balance the efficient Förster energy transfer for giving pure emission of (DT) ${ }_{2} \mathbf{B T h} \mathbf{2} \mathbf{C N}$ and the competitive quenching process by the inevitable Dexter energy transfer, $10 \mathrm{wt} \%$ (DT) ${ }_{2} \mathbf{B T h} 2 \mathrm{CN}$ was selected as the doping concentration for the exciplex cohosts (Tr-Ph/Tr-Tol:3P-T2T and Tr-Ph/Tr-Tol:3P-T2P) to investigate the performance of the deep-red fluorescent devices. Fig. 6(b-d) depict the EL characteristics of these deep-red fluorescent devices, and the key data are summarized in Table 3. All $10 \mathrm{wt} \%$ (DT) $)_{2} \mathbf{B T h} 2 \mathbf{C N}$-doped devices exhibit pure emission from the dopant with the EL peak centered at $c a .670 \mathrm{~nm}$. Among them, the Tr-Ph:3P-T2P-hosted device A2r shows the best performance, achieving maximum EQE of $5.52 \%$, CE of $1.66 \mathrm{~cd} \mathrm{~A}^{-1}$ and PE of $2.09 \mathrm{~lm} \mathrm{~W}^{-1}$ with CIE coordinates of $(0.68,0.31)$. The $5.52 \%$ EQE based on a Tr-Ph:3P-T2T cohost is higher than the theoretical limit of $5 \%$ typically anticipated for a conventional fluorescence emitter. The device performances are in good agreement with the
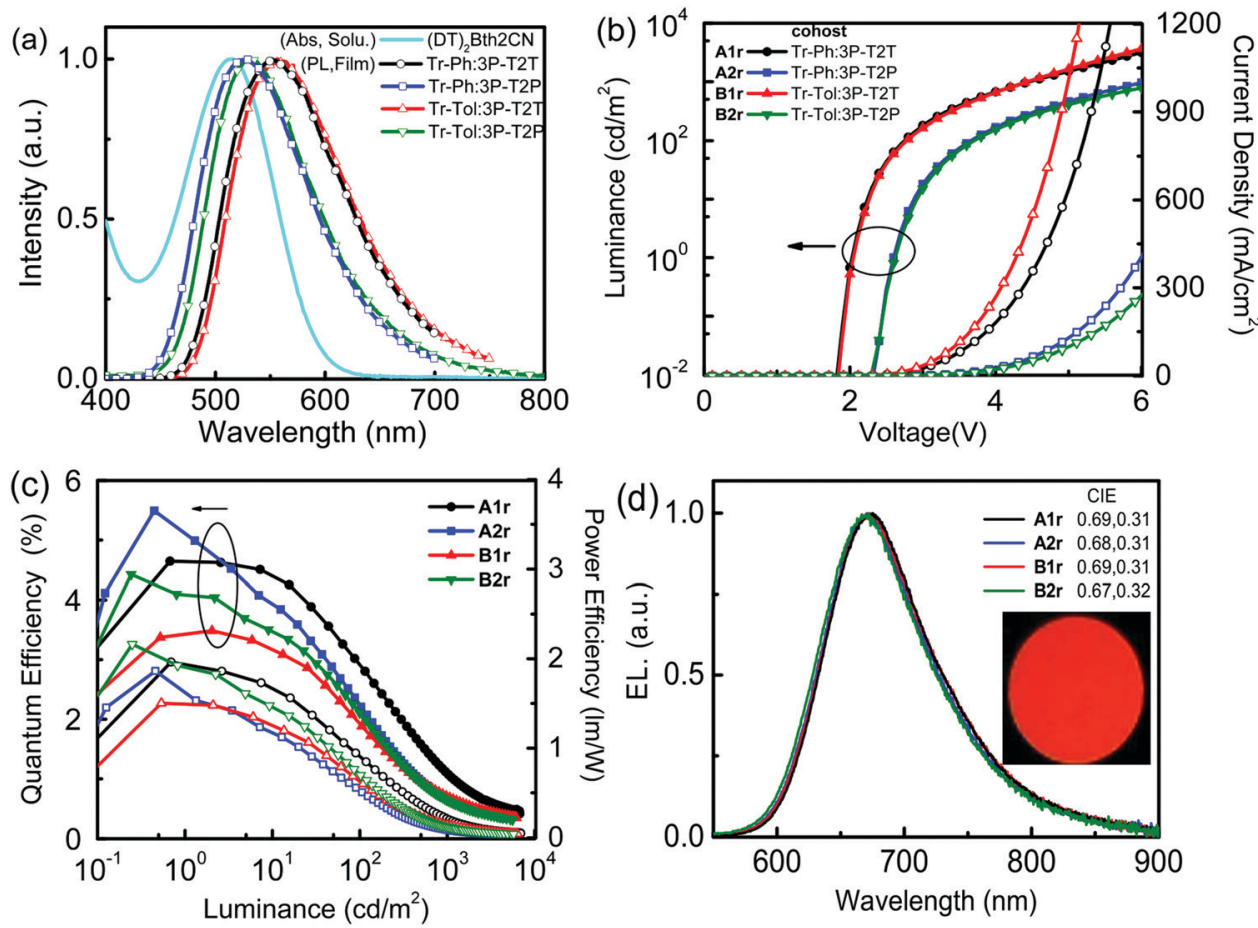

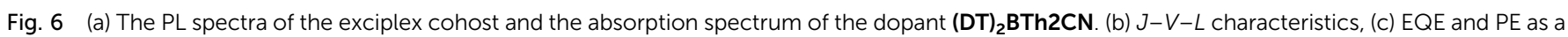
function of luminance and (d) EL spectra of $10 \mathrm{wt} \%(\text { DT })_{2} \mathbf{B T h} \mathbf{2 C N}$ doped devices. 
PLQYs of the (DT) ${ }_{2} \mathbf{B T h} 2 \mathbf{C N}$ emission extracted from the exciplexforming cohosts as in the data summarized in Table S2 (ESI $\dagger$ ). TRPL measurements were further performed on the (DT) $)_{2} \mathbf{B T h} 2 \mathrm{CN}$-doped exciplex cohosts (Tr-Ph/Tr-Tol:3P-T2T and Tr-Ph/Tr-Tol:3P-T2P) to probe the working mechanism. The data are summarized in Table S2 (ESI $\dagger$ ). As indicated, the (DT) ${ }_{2} \mathbf{B T h} 2 \mathbf{C N}$-dispersed films show a dominant prompt decay component with a lifetime of around $7 \mathrm{~ns}$ together with a delay component with a lifetime of around $0.13 \mu \mathrm{s}$. The delay component only counted for about $1 \%$ of the whole relaxation process when $\mathbf{T r}$-Ph was applied as the donor in the blended film. In the case of Tr-Tol utilized as the donor for exciplex-forming systems, the delay components are as low as only $0.2 \%$ of the overall relaxation process. Accordingly, the EL from the exciplex-forming cohosts doped with 10 wt\% (DT) $)_{2} \mathbf{B T h} 2 \mathbf{C N}$ is mainly contributed by the Förster energy transfer, but simultaneously suffers from the inferior triplet-harvesting of the exciplex due to the competing Dexter energy transfer quenching in the presence of high dopant concentration.

\section{Conclusion}

For the first time, three triazatruxenes (Tr-Me, Tr-Ph, Tr-Tol) were examined as donors for exciplex formation with $\mathrm{N}$-heteroarene-cored acceptors (3P-T2T, 3P-T2P, 3P-Pyr). As compared to those of pristine donor and acceptor films, these nine D:A combinations were found to show red-shifted emissions that are the signatures of forming exciplexes. The emission energies of the exciplexes corresponding to the interfacial energy difference between the HOMO (donor) and the LUMO (acceptor) are governed mainly by the features of $\mathrm{N}$-heteroarene cores of acceptors, while the HOMOs of triazatruxene donors are less sensitive to the substituents. The red-shifted absorption and emission spectra of Tr-Me pristine film indicate the evident intermolecular interactions due to the less hindered methyl substitutions. The severe self-aggregation together with the coplanar feature of acceptors prevent the complete exciplex formation of Tr-Me:3P-T2T and Tr-Me:3P-T2P blends, as indicated by the presence of the residual emission of Tr-Me. The inferior exciplex formation of Tr-Me can be suppressed by combining the acceptor 3P-Pyr with twisted conformation. To our surprise, the blends using 3P-Pyr as acceptor and Tr-Ph, Tr-Tol as the respective donors gave low PLQYs due to the insufficient polarization effect for promoting efficient rISC. Systematic examination of the device characteristics concluded that the four blends composed of Tr-Ph and Tr-Tol as donors and 3P-T2T and 3P-T2P as acceptors are good EMLs for OLED applications. Among them, the Tr-Tol:3P-T2P blend gave a high-efficiency green device with an $\mathrm{EQE}_{\max }$ of up to $12.8 \%$, agreeing with the observed superior PLQY upon photoexcitation. These new exciplex-forming systems were further exploited as cohosts to fabricate deep-red devices with a tailormade fluorescent dopant $(\mathbf{D T})_{\mathbf{2}} \mathbf{B T h} 2 \mathbf{C N}$. The deep-red device based on the $10 \mathrm{wt} \%$ (DT) ${ }_{2} \mathbf{B T h 2 C N}$ doped Tr-Ph:3P-T2P blend as the EML gave EL $\lambda_{\max } 671 \mathrm{~nm}$ and CIE $(0.68,0.31)$ solely from the dopant and an $\mathrm{EQE}_{\max }$ of $5.52 \%$, which is consistent with the highest PLQY observed in the exciplex-hosted (DT) ${ }_{2} \mathbf{B T h} 2 \mathbf{C N}$ films. The TRPL studies on the exciplex-hosted (DT) $)_{2} \mathbf{B T h} 2 \mathbf{C N}$ films provide information for proposing a reasonable working mechanism in an EL device. The limited proportion of the delayed component in the whole relaxation process of $(\mathbf{D T})_{2} \mathbf{B T h} 2 \mathbf{C N}$ doped in exciplex-forming blends implies that the EL is mainly contributed by the singlet-to-singlet Förster energy transfer without the aid of triplet-harvesting from the exciplex cohost due to the competitive triplet-to-triplet quenching of Dexter energy transfer occurring in the presence of a high dopant concentration. Our results reveal that the characteristics of exciplex-forming systems can be feasibly tuned by systematic structural variations implemented on donor and/or acceptor components and the versatile functions of an exciplex can be achieved in OLED applications.

\section{Conflicts of interest}

There are no conflicts to declare.

\section{Acknowledgements}

The authors acknowledge financial support from the Ministry of Science and Technology (Grant No. MOST 107-2113-M-002019-MY3 and 106-2112-M-019-002-MY3).

\section{References}

1 A. Endo, M. Ogasawara, A. Takahashi, D. Yokoyama, Y. Kato and C. Adachi, Thermally activated delayed fluorescence from $\mathrm{Sn}^{4+}$-porphyrin complexes and their application to organic light emitting diodes-a novel mechanism for electroluminescence, Adv. Mater., 2009, 21, 4802-4806.

2 M. Ikai and S. Tokito, Highly efficient phosphorescence from organic light-emitting devices with an exciton-block layer, Appl. Phys. Lett., 2001, 79, 156-158.

3 A. Endo, K. Sato, K. Yoshimura, T. Kai, A. Kawada, H. Miyazaki and C. Adachi, Efficient up-conversion of triplet excitons into a singlet state and its application for organic light emitting diodes, Appl. Phys. Lett., 2011, 98, 083302.

4 K. Kawasumi, T. Wu, T. Zhu, H. S. Chae, T. V. Voorhis, M. A. Baldo and T. M. Swager, Thermally activated delayed fluorescence materials based on homoconjugation effect of donor-acceptor triptycenes, J. Am. Chem. Soc., 2015, 137, 11908-11911.

5 H. Kaji, H. Suzuki, T. Fukushima, K. Shizu, K. Suzuki, S. Kubo, T. Komino, H. Oiwa, F. Suzuki, A. Wakamiya, Y. Murata and C. Adachi, Purely organic electroluminescent material realizing $100 \%$ conversion from electricity to light, Nat. Commun., 2015, $6,8476$.

6 H. Uoyama, K. Goushi, K. Shizu, H. Nomura and C. Adachi, Highly efficient organic light-emitting diodes from delayed fluorescence, Nature, 2012, 492, 234-238.

7 T.-L. Wu, M.-J. Huang, C.-C. Lin, P.-Y. Huang, T.-Y. Chou, R.-W. Cheng, H.-W. Lin, R.-S. Liu and C.-H. Cheng, Diboron compound-based organic light-emitting diodes with high 
efficiency and reduced efficiency roll-off, Nat. Photonics, 2018, 12, 235-240.

8 D. Zhang, M. Cai, Z. Bin, Y. Zhang, D. Zhang and L. Duan, Highly efficient blue thermally activated delayed fluorescent OLEDs with record-low driving voltages utilizing high triplet energy hosts with small singlet-triplet splittings, Chem. Sci., 2016, 7, 3355-3363.

9 W. Zeng, H.-Y. Lai, W.-K. Lee, M. Jiao, Y.-J. Shiu, C. Zhong, S. Gong, T. Zhou, G. Xie, M. Sarma, K.-T. Wong, C.-C. Wu and C. Yang, External quantum efficiency for orange-red organic light emitting diodes by employing thermally activated delayed fluorescence emitters composed of 1,8-naphthalimideacridine hybrids, Adv. Mater., 2018, 30, 1704961.

10 S.-W. Li, C.-H. Yu, C.-L. Ko, T. Chatterjee, W.-Y. Hung and K.-T. Wong, Cyanopyrimidine-carbazole hybrid host materials for high-efficiency and low-efficiency roll-off TADF OLEDs, ACS Appl. Mater. Interfaces, 2018, 10, 12930-12936.

11 X.-K. Liu, Z. Chen, C.-J. Zheng, M. Chen, W. Liu, X.-H. Zhang and C.-S. Lee, Nearly $100 \%$ triplet harvesting in conventional fluorescent dopant-based organic light-emitting devices through energy transfer from exciplex, Adv. Mater., 2015, 27, 2025-2030.

12 K. Goushi and C. Adachi, Efficient organic light-emitting diodes through up-conversion from triplet to singlet excited states of exciplexes, Appl. Phys. Lett., 2012, 101, 023306.

13 V. Jankus, C.-J. Chiang, F. Dias and A. P. Monkman, Deep blue exciplex organic light-emitting diodes with enhanced efficiency; p-type or e-type triplet conversion to singlet excitons?, Adv. Mater., 2013, 25, 1455-1459.

14 B. S. Kim and J. Y. Lee, Engineering of mixed host for high external quantum efficiency above $25 \%$ in green thermally activated delayed fluorescence device, Adv. Funct. Mater., 2014, 24, 3970-3977.

15 M. Zhang, K. Wang, C.-J. Zheng, D.-Q. Wang, Y.-Z. Shi, H. Lin, S.-L. Tao, X. Li and X.-H. Zhang, Development of red exciplex for efficient OLEDs by employing a phosphor as a component, Front. Chem., 2019, 7, 16.

16 Y. Hu, Y.-J. Yu, Y. Yuan, Z.-Q. Jiang and L.-S. Liao, Exciplexbased organic light-emitting diodes with near-infrared emission, Adv. Opt. Mater., 2020, 1901917.

17 T. Zhang, B. Zhao, B. Chu, W. Li, Z. Su, X. Yan, C. Liu, H. Wu, Y. Gao, F. Jin and F. Hou, Simple structured hybrid WOLEDs based on incomplete energy transfer mechanism: from blue exciplex to orange dopant, Sci. Rep., 2015, $5,10234$.

18 S. Lee, K.-H. Kim, D. Limbach, Y.-S. Park and J.-J. Kim, Low roll-off and high efficiency orange organic light emitting diodes with controlled co-doping of green and red phosphorescent dopants in an exciplex forming co-host, Adv. Funct. Mater., 2013, 23, 4105-4110.

19 M. Sarma and K.-T. Wong, Exciplex: an intermolecular charge-transfer approach for TADF, ACS Appl. Mater. Interfaces, 2018, 10, 19279-19304.

20 N. R. A. Amin, K. K. Kesavan, S. Biring, C.-C. Lee, T.-H. Yeh, T.-Y. Ko, S.-W. Liu and K.-T. Wong, A comparative study via photophysical and electrical characterizations on interfacial and bulk exciplex-forming systems for efficient organic lightemitting diodes, ACS Appl. Electron. Mater., 2020, 2, 1011-1019.

21 M. Guzauskas, D. Volyniuk, A. Tomkeviciene, A. Pidluzhna, A. Lazauskasc and J. V. Grazulevicius, Dual nature of exciplexes: exciplex-forming properties of carbazole and fluorene hybrid trimers, J. Mater. Chem. C, 2019, 7, 25-32.

22 S. W. Shelton, T. L. Chen, D. E. Barclay and B. Ma, Solutionprocessable triindoles as hole selective materials in organic solar cells, ACS Appl. Mater. Interfaces, 2012, 4, 2534-2540.

23 D. H. Ahn, S. W. Kim, H. Lee, I. J. Ko, D. Karthik, J. Y. Lee and J. H. Kwon, Highly efficient blue thermally activated delayed fluorescence emitters based on symmetrical and rigid oxygen-bridged boron acceptors, Nat. Photonics, 2018, 13, 540-546.

24 K. Rakstys, A. Abate, M. I. Dar, P. Gao, V. Jankauskas, G. Jacopin, E. Kamarauskas, S. Kazim, S. Ahmad, M. Grätzel and M. K. Nazeeruddin, Triazatruxene-based hole transporting materials for highly efficient perovskite solar cells, J. Am. Chem. Soc., 2015, 137, 16172-16178.

25 S. Mula, T. Han, T. Heiser, P. Lévêque, N. Leclerc, A. P. Srivastava, A. R. Carretero and G. Ulrich, Hydrogen bonding as a supramolecular tool for robust OFET devices, Chem. - Eur. J., 2019, 25, 8304-8312.

26 J.-H. Lee, S.-H. Cheng, S.-J. Yoo, H. Shin, J.-H. Chang, C.-I. Wu, K.-T. Wong and J.-J. Kim, An exciplex forming host for highly efficient blue organic light emitting diodes with low driving voltage, Adv. Funct. Mater., 2015, 25, 361.

27 H.-F. Chen, T.-C. Wang, S.-W. Lin, W.-Y. Hung, H.-C. Dai, H.-C. Chiu, K.-T. Wong, M.-H. Ho, T.-Y. Cho, C.-W. Chen and C.-C. Lee, Peripheral modification of 1,3,5-triazine based electron-transporting host materials for sky blue, green, yellow, red, and white electrophosphorescent devices, J. Mater. Chem., 2012, 22, 15620-15627.

28 G. W. Kim, R. Lampande, D. C. Choe, H. W. Bae and J. H. Kwon, Efficient hole injection material for low operating voltage blue fluorescent organic light emitting diodes, Thin Solid Films, 2015, 589, 105-110.

29 D. H. Huh, G. W. Kim, G. H. Kim, C. Kulshreshtha and J. H. Kwon, High hole mobility hole transport material for organic light-emitting devices, Synth. Met., 2013, 180, 79-84.

30 M. Franceschin, L. G. Satriani, A. Alvino, G. Ortaggi and A. Bianco, Study of a convenient method for the preparation of hydrosoluble fluorescent triazatruxene derivatives, Eur. J. Org. Chem., 2010, 134-141.

31 S.-J. Su, C. Cai and J. Kido, Three-carbazole-armed host materials with various cores for RGB phosphorescent organic light-emitting diodes, J. Mater. Chem., 2012, 22, 3447-3456.

32 A. G. Martinez, A. H. Fernandez, F. M. Jimenez, A. G. Fraile, L. R. Subramanian and M. Hanack, On the mechanism of the reaction between ketones and trifluoromethanesulfonic anhydride. An improved and convenient method for the preparation of pyrimidines and condensed pyrimidines, J. Org. Chem., 1992, 57, 1627-1630.

33 J. D. Douglas, G. Griffini, T. W. Holcombe, E. P. Young, O. P. Lee, M. S. Chen and J. M. J. Fréchet, Functionalized 
isothianaphthene monomers that promote quinoidal character in donor-acceptor copolymers for organic photovoltaics, Macromolecules, 2012, 45, 4069-4074.

34 S.-J. Su, H. Sasabe, T. Takeda and J. Kido, Pyridinecontaining bipolar host materials for highly efficient blue phosphorescent OLEDs, Chem. Mater., 2008, 20, 1691-1693.

35 Y. Watanabe, D. Yokoyama, T. Koganezawa, H. Katagiri, T. Ito, S. Ohisa, T. Chiba, H. Sasabe and J. Kido, Control of molecular orientation in organic semiconductor films using weak hydrogen bonds, Adv. Mater., 2019, 31, 1808300.

36 M. Wang, Y.-H. Huang, K.-S. Lin, T.-H. Yeh, J. Duan, T.-Y. Ko, S.-W. Liu, K.-T. Wong and B. Hu, Revealing the cooperative relationship between spin, energy, and polarization parameters toward developing high-efficiency exciplex light-emitting diodes, Adv. Mater., 2019, 31, 1904114.

37 D.-S. Leem, H.-D. Park, J.-W. Kang, J.-H. Lee, J. W. Kim and J.-J. Kim, Low driving voltage and high stability organic light-emitting diodes with rhenium oxide-doped hole transporting layer, Appl. Phys. Lett., 2007, 91, 011113.

38 W.-Y. Hung, P.-Y. Chiang, S.-W. Lin, W.-C. Tang, Y.-T. Chen, S.-H. Liu, P.-T. Chou, Y.-T. Hung and K.-T. Wong, Balance the carrier mobility to achieve high performance exciplex OLED using a triazine-based acceptor, ACS Appl. Mater. Interfaces, 2016, 8, 4811-4818.
39 C.-J. Shih, C.-C. Lee, Y.-H. Chen, S. Biring, G. Kumar, T.-H. Yeh, S. Sen, S.-W. Liu and K.-T. Wong, Exciplex-forming cohost for high efficiency and high stability phosphorescent organic light emitting diodes, ACS Appl. Mater. Interfaces, 2018, 10, 2151-2157.

40 W.-Y. Hung, G.-C. Fang, Y.-C. Chang, T.-Y. Kuo, P.-T. Chou, S.-W. Lin and K.-T. Wong, Highly efficient bilayer interface exciplex for yellow organic light-emitting diode, ACS Appl. Mater. Interfaces, 2013, 5, 6826-6831.

41 Y.-C. Lo, T.-H. Yeh, C.-K. Wang, B.-J. Peng, J.-L. Hsieh, C.-C. Lee, S.-W. Liu and K.-T. Wong, High-efficiency red and nearinfrared organic light-emitting diodes enabled by pure organic fluorescent emitters and an exciplex-forming cohost, ACS Appl. Mater. Interfaces, 2019, 11, 23417-23427.

42 Q. Wang, Q.-S. Tian, Y.-L. Zhang, X. Tang and L.-S. Liao, High-efficiency organic light-emitting diodes with exciplex hosts, J. Mater. Chem. C, 2019, 7, 11329-11360.

43 B. Zhao, T. Zhang, B. Chu, W. Li, Z. Su, H. Wu, X. Yan, F. Jin, Y. Gao and C. Liu, Highly efficient red OLEDs using DCJTB as the dopant and delayed fluorescent exciplex as the host, Sci. Rep., 2015, 5, 10697.

44 K.-H. Kim, C.-K. Moon, J. W. Sun, B. Sim and J.-J. Kim, Triplet harvesting by a conventional fluorescent emitter using reverse intersystem crossing of host triplet exciplex, Adv. Opt. Mater., 2015, 3, 895-899. 